\title{
Role of Mean Arterial Pressure in Mid-trimester Pregnancy for the Prediction of Gestational Hypertension and Pre-eclampsia
}

\author{
Ojasvi Shanker ${ }^{1}$, Mamta Gupta ${ }^{2}$
}

\begin{abstract}
Background: Reliable markers for the prediction of pre-eclampsia (PE) and reducing its associated maternal and perinatal morbidity are lacking. Aims and objectives: To evaluate the role of mean arterial pressure (MAP) in the second trimester of pregnancy for predicting gestational hypertension (GH) and PE.

Materials and Methods: Three-hundred and sixteen healthy and normotensive women were enrolled in the second trimester of pregnancy. The mean MAP for the woman was recorded as an average of two MAPs at 3-4 week intervals during the second trimester of pregnancy. All women were followed till term/delivery to predict the development of $\mathrm{GH}$ and pre-eclampsia later.

Results: The performance of MAP for predicting the GH and PE was found to be very good. The area under the receiver operating characteristic (AUROC) for GH was 0.892 with sensitivity and specificity of 84.2 and $84.9 \%$, respectively, whereas the AUROC for PE was 0.948 with sensitivity and specificity of 83.3 and $84.9 \%$, respectively.

Conclusion: MAP in the second trimester of pregnancy can be used to triage women with low-risk pregnancy for pregnancy hypertension.

Clinical significance: Mid-trimester MAP is a very good parameter for the prediction of GH and PE. It should be routinely used for risk triaging in low-risk women for the development of hypertension in pregnancy.

Keywords: Gestational hypertension, Mean arterial pressure, Prediction of pre-eclampsia, Pre-eclampsia.

Journal of South Asian Federation of Obstetrics and Gynaecology (2021): 10.5005/jp-journals-10006-1898
\end{abstract}

\section{INTRODUCTION}

Prediction of gestational hypertension $(\mathrm{GH})$ and pre-eclampsia (PE) has been a challenge for the obstetrician. It is the most common medical complication of pregnancy, occurring in 2 to $8 \%$ of all pregnancies. ${ }^{1}$ Pregnant women with these hypertensive conditions are at higher risk for severe complications and maternal-fetal morbidity and mortality. Maternal morbidity includes renal failure, stroke, cardiac dysfunction or arrest, respiratory compromise, coagulopathy, and liver failure. ${ }^{2,3}$ The fetus may become hypoxic, increasing the risk of low birth weight, premature delivery, and perinatal death. ${ }^{2}$ In a recent study, it was shown that hypertensive disorders were responsible for $15.77 \%$ of all stillbirths. ${ }^{4}$ The only effective treatment for pregnancy hypertension is delivery of the placenta. As the disease etiology remains largely unclear, there is little role of primary prevention. Secondary level prevention is more significant, which means diagnosing the disease process at its earliest and providing timely and appropriate management for this disease. Predicting PE in early pregnancy in women at risk of PE may decrease maternal and fetal morbidity. Investigators have been evaluating tests to predict PE over the last few decades without much success. ${ }^{5,6}$ These have included tests relating to placental perfusion and vascular resistance (e.g., the "roll over" and cold pressor tests, uterine artery Doppler evaluation); placental products (e.g., pro- and antiangiogenic proteins, human chorionic gonadotropin, placental protein 13, and inhibin A); renal dysfunction (e.g., fractional urate clearance and microalbuminuria); and endothelial dysfunction (e.g., fibronectin, P- and L-selectin, and VCAM-1). Meads et al. reviewed 27 tests for the prediction of PE. ${ }^{7}$ However, only a few reached a specificity of $90 \%$, and no single test met the clinical standards for a predictive test. ${ }^{6}$
${ }^{1}$ Department of Obstetrics and Gynaecology, Manipal Tata Medical College, Jamshedpur, Jharkhand, India

${ }^{2}$ Department of Obstetrics and Gynaecology, Hindu Rao Hospital, Delhi, India

Corresponding Author: Ojasvi Shanker, Department of Obstetrics and Gynaecology, Manipal Tata Medical College, Jamshedpur, Jharkhand, India, Phone: +91 9311840325, e-mail: ojasvishanker@gmail.com

How to cite this article: Shanker O, Gupta M. Role of Mean Arterial Pressure in Mid-trimester Pregnancy for the Prediction of Gestational Hypertension and Pre-eclampsia. J South Asian Feder Obst Gynae 2021;13(3):151-155.

Source of support: Nil

Conflict of interest: None

In uncomplicated pregnancies, the arterial blood pressure pattern usually consists of a steady decrease in blood pressure (BP) during the first half of the pregnancy and then increases until the time of delivery. ${ }^{8}$ In contrast, in women with hypertensive disorder (GH or PE), BP is generally stable during the first half of the pregnancy and then increases until delivery.

It has been shown that women destined to develop PE have higher mean arterial pressures (MAP) in the first and second trimesters than women with normal pregnancies. ${ }^{9}$ It is the average arterial pressure during a single cardiac cycle. MAP is a possible indicator of cardiovascular adaptations in pregnancy. It is calculated by DBP + onethird of the pulse pressure. A decreased peripheral resistance leads to a decline in BP and also MAP in the first half of the pregnancy.

Thus, MAP screening provides an important adjunctive tool for the early identification of women at increased risk of obstetrical 
complications and adverse fetal outcome. These women constitute the group of high-risk pregnancies who require intensive antenatal care (ANC).

\section{Aim and Objects}

To evaluate the role of MAP in the second trimester of pregnancy for predicting $\mathrm{GH}$ and $\mathrm{PE}$.

\section{Materials and Methods}

All pregnant women attending the antenatal clinic at Northern Railway Central Hospital, New Delhi, India, with their first visit at or before 20 weeks of gestation between May 1, 2012 and April 30, 2014, were enrolled after obtaining the written consent and fulfilling the inclusion and exclusion criteria.

\section{Inclusion Criteria}

Women with singleton pregnancy of 14-20 weeks were included in this study (calculated by last menstrual period or first trimester ultrasonography).

\section{Exclusion Criteria}

Women with disorders complicating pregnancy, that is, essential hypertension, heart disease, renal disease, diabetes, hydramnios, twins, and Rh-negative pregnancy, were excluded.

The study was approved by the Institutional Ethics Committee. In this prospective longitudinal study, 316 pregnant women fulfilling inclusion and exclusion criteria were enrolled and evaluated. A detailed history was taken. General, systemic, and obstetric examination was done. BP was taken twice at an interval of 1 minute in both arms in sitting position after resting for 5 minutes, with an arm held at the level of the heart. The mean of the two $B P$ readings taken was used to calculate the MAP. MAP was calculated using the following formula from the mean of the four readings:

$\mathrm{MAP}=\mathrm{DBP}+1 / 3$ pulse pressure $\mathrm{OR}$

$M A P=D B P+1 / 3(S B P-D B P)$

Two MAP values, 3-4 weeks apart between 16 and 26 weeks (mid-trimester) of gestation, were taken. An average for both the MAP readings was calculated and recorded as mid-trimester MAP of the woman.

Proteinuria was assessed by dipstick. A reading of $1+$ proteinuria in dipstick was considered significant proteinuria. Women were followed up in the antenatal outpatient department for every 3-4 weeks till 34 weeks and then 1 to 2 weekly till the term / delivery for the development of GH or PE. BP measurement and test for proteinuria was done at every scheduled visit. Women who could not be followed up till term were excluded from the study. A total of 316 women were followed till delivery/ term.

\section{Statistical Analysis}

Statistical analysis was performed by the SPSS program for Windows, version 17.0 (SPSS, Chicago, Illinois). The tests used to analyze the data were the Chi-square test and analysis of variance test. A receiver operating characteristic $(\mathrm{ROC})$ analysis was calculated to determine the optimal cutoff values of MAP for predicting the $\mathrm{GH}$ and $P E$, and sensitivity, specificity, positive predictive value (PPV), and negative predictive value (NPV) were calculated accordingly. For all statistical tests, a $p$ value less than 0.05 was taken to indicate a significant difference.

\section{Results}

Three-hundred and sixteen women who were followed up till term/delivery were divided into three groups depending on the development or nondevelopment of GH or PE.

- Group A comprised of women who remained normotensive $(n=285)$.

- Group B comprised of women who developed GH (defined as

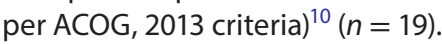

- Group C comprised of women who developed PE (defined as per ACOG, 2013 criteria $)^{10}(n=12)$.

The area under the curve (AUC) for the prediction of $\mathrm{GH}$ was estimated to be 0.892 . The cutoff for predicting the $\mathrm{GH}$ was estimated to be $89.17 \mathrm{~mm} \mathrm{Hg}$ from the ROC (Fig. 1). Using this cutoff, the number of women who developed and who did not develop $\mathrm{GH}$ was found to be highly significant ( $p$-value, 0.001).

The AUC for the prediction of PE was estimated to be 0.942 . The cutoff for predicting PE was estimated to be $93.67 \mathrm{~mm} \mathrm{Hg}$ from the ROC (Fig. 2). Using this cutoff, the number of women who developed and who did not develop PE was found to be highly significant ( $p$-value, 0.001).

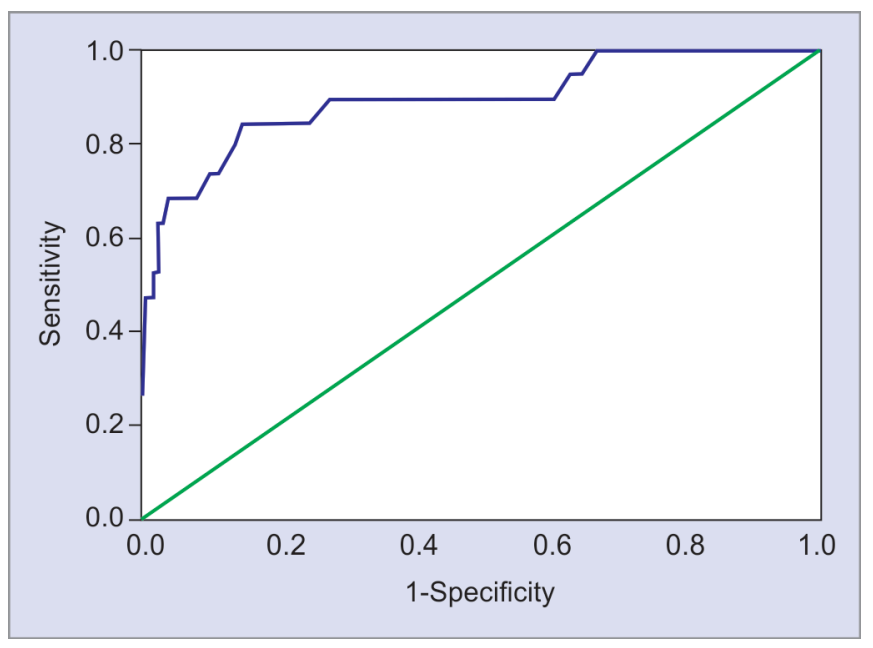

Fig. 1: ROC curve for GH

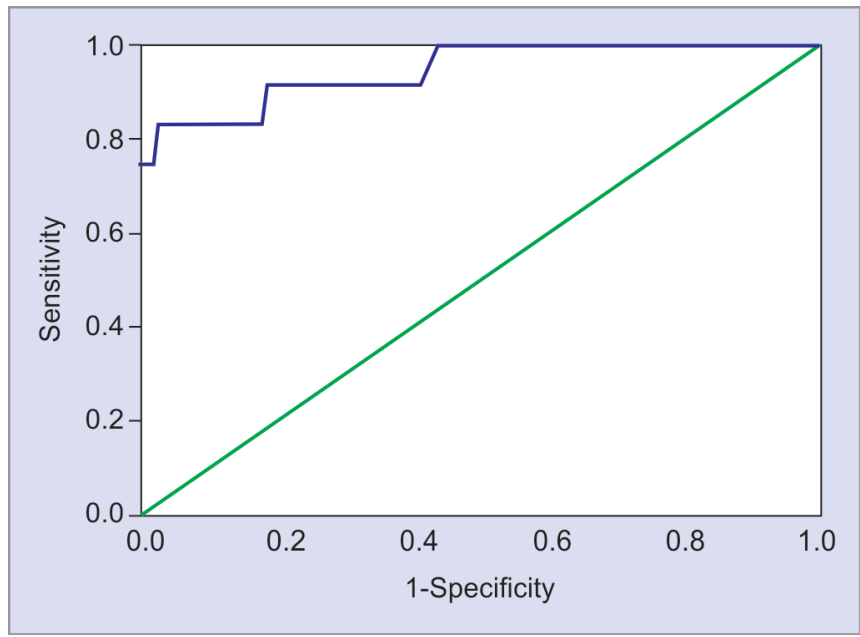

Fig. 2: ROC curve for PE 


\section{Discussion}

In this study, 316 pregnant women were enrolled and analyzed. Two-hundred and eighty-five women remained normotensive during follow-up. Nineteen women developed $\mathrm{GH}$, and 12 women developed $\mathrm{PE}$, making the incidence of $\mathrm{GH}$ and $\mathrm{PE}$ as $6.66 \%$ and $4.21 \%$, respectively. Umegbolu El et al. in their study of 10 years (2005-2016) have reported a similar incidence of GH as 5.9\%. ${ }^{11}$ However, Rajesh et al. and Dawle et al. have found a much higher incidence of pregnancy-induced hypertension, that is,14.8 and 20\%, respectively, in their studies. ${ }^{12,13}$

The age of the women in the three groups was found to be comparable. The majority of the women who developed GH were in the age-group of 20-25 years (47.36\%). However, Umegbolu EI et al. ${ }^{11}$ showed that women above 35 years of age had a significant high incidence of hypertensive disease of pregnancy.

Various maternal parameters like parity and mean midtrimester MAP were found to be significantly high in groups B and $C$ compared to $A$ (Table 1). The mean mid-trimester MAP was significantly high at all the ranges in women who subsequently developed GH or PE ( $p$-value, 0.001 ) (Table 1). None of the patients having MAP in the range of $70-79.9$ developed GH or PE. The maximum number of patients developing GH (36.8\%) had a MAP in the range of $95-99.9 \mathrm{~mm} \mathrm{Hg}$, and those developing PE $(41.7 \%)$ had a MAP greater than $100 \mathrm{~mm}$ Hg. Similar findings were reported by Miller et al. ${ }^{14}$ They classified participants by MAP quartiles: less than $79,79-83,84-88$, and greater than or equal to $89 \mathrm{~mm} \mathrm{Hg}$ and reported that high-quartile MAP was associated with an increased risk of PE. ${ }^{14}$
The AUC for the prediction of GH and PE was found to be 0.892 and 0.948 , respectively, in our study showing that MAP has a strong association with the development of GH or PE and can be used for the prediction of $\mathrm{GH}$ and $\mathrm{PE}$, more so for $\mathrm{PE}$.

Using ROC, the optimal cutoff value of the mean mid-trimester MAP for GH was found to be $89.17 \mathrm{~mm} \mathrm{Hg}$. Out of 19 women who developed $\mathrm{GH}$, only three women had mid-trimester MAP below this cut-off value. Thus, the chance of developing GH was significantly high in women with MAP greater than $89.17 \mathrm{~mm} \mathrm{Hg}(n=16)$ than in women with MAP less than $89.17 \mathrm{~mm} \mathrm{Hg}$ ( $p$-value, 0.001).

Similarly, in our study, the optimal cutoff value of MAP for diagnosing PE was found to be $93.67 \mathrm{~mm} \mathrm{Hg}$ using ROC (Fig. 2). A significantly more number of women developed PE who had MAP greater than $93.67 \mathrm{~mm} \mathrm{Hg}$ in mid-trimester of pregnancy $(n=10)$ compared to those with MAP less than $93.67 \mathrm{~mm} \mathrm{Hg}(n=2)$ ( $p$-value, 0.001).

Mayrink et al. have reported that MAP performance was modest as a predictor, with an AUC of only $0.619 .{ }^{15}$ However, a systematic review from 2008 demonstrated that mean MAP measured during the first or second trimester of gestation in a general low-risk pregnant population is a better predictor of PE with AUC of $0.76 .^{16}$

We observed that mean MAP was not significantly associated with the timing of onset of GH or PE (early/late onset) (Table 2) ( $p$-value, 0.437 and 0.301 , respectively) or with the severity of PE (with or without severe features) ( $p$-value, 0.95 ) (Table 3). However, Mayrink et al. have shown that in the early-onset PE group, MAP showed the highest value at 20 weeks of gestation, compared to the control group $(p$-value $=0.024) .^{15}$

Table 1: Baseline data $(N=316)$

\begin{tabular}{|c|c|c|c|c|}
\hline Variable & $\begin{array}{l}\text { Normotensive } \\
n=285(\%)\end{array}$ & $\begin{array}{l}\text { GH group } \\
n=19(\%)\end{array}$ & $\begin{array}{l}\text { PE group } \\
n=12(\%)\end{array}$ & $p$ value \\
\hline \multicolumn{5}{|l|}{ Age (years) } \\
\hline$<20$ & $2(0.70 \%)$ & $0(0 \%)$ & $0(0 \%)$ & 0.539 \\
\hline $20-25$ & 115 (40.35\%) & $9(47.36 \%)$ & $3(25 \%)$ & \\
\hline $26-30$ & $128(44.91 \%)$ & $5(26.31 \%)$ & $5(41.66 \%)$ & \\
\hline $31-35$ & $32(11.22 \%)$ & $4(21.05 \%)$ & $3(25 \%)$ & \\
\hline$>36$ & $8(2.80 \%)$ & $1(5.26 \%)$ & $1(8.33 \%)$ & \\
\hline Mean age (years) & $26.66 \pm 3.97$ & $27.26 \pm 4.87$ & $29.00 \pm 4.02$ & 0.127 \\
\hline \multicolumn{5}{|l|}{ Parity } \\
\hline Primi & 88 (30.88\%) & $13(68.42 \%)$ & $5(41.67 \%)$ & 0.003 \\
\hline Multi & 197 (69.12\%) & $6(31.57 \%)$ & 7 (58.33\%) & \\
\hline \multicolumn{5}{|l|}{ MAP range $(\mathrm{mm} \mathrm{Hg})$} \\
\hline $70-74.9$ & $13(4.56 \%)$ & $0(0 \%)$ & $0(0 \%)$ & 0.001 \\
\hline $75-79.9$ & $46(16.14 \%)$ & $0(0 \%)$ & $0(0 \%)$ & \\
\hline $80-84.9$ & 109 (38.24\%) & $2(10.52 \%)$ & $1(8.33 \%)$ & \\
\hline $85-89.9$ & $84(29.47 \%)$ & $3(15.79 \%)$ & $1(8.33 \%)$ & \\
\hline $90-94.9$ & $27(9.47 \%)$ & $5(26.31 \%)$ & $1(8.33 \%)$ & \\
\hline $95-99.9$ & $6(2.10 \%)$ & $7(24.56 \%)$ & $4(33.33 \%)$ & \\
\hline$>100$ & $0(0 \%)$ & $2(10.52 \%)$ & $5(41.67 \%)$ & \\
\hline \multicolumn{5}{|c|}{ Mean second trimester } \\
\hline $\mathrm{MAP}, \mathrm{mm} \mathrm{Hg}$ & $83.82 \pm 5.18$ & $94.00 \pm 6.31$ & $97.33 \pm 5.66$ & 0.001 \\
\hline \multicolumn{5}{|c|}{$\begin{array}{l}\text { Mean BP at diagnosis of } \\
\text { GH/PE }\end{array}$} \\
\hline Systolic (mm Hg) & $114.21 \pm 8.15$ & $145.89 \pm 3.86$ & $152.50 \pm 6.61$ & 0.001 \\
\hline Diastolic $(\mathrm{mm} \mathrm{Hg})$ & $76.28 \pm 5.45$ & $93.68 \pm 3.90$ & $100.83 \pm 5.15$ & 0.001 \\
\hline
\end{tabular}


Table 2: Association of MAP with early- and late-onset GH and PE

\begin{tabular}{llrrl}
\hline Group & $P O G$ (weeks) & $n(\%)$ & Mean MAP $\pm S D$ & $p$ value \\
\hline $\mathrm{GH}$ & $<34$ & $4(21.05 \%)$ & $96.253 \pm 10.403$ & 0.437 \\
$(N=19)$ & $\geq 34$ & $15(78.95 \%)$ & $93.401 \pm 5.109$ & \\
\multirow{2}{*}{$\mathrm{PE}$} & $<34$ & $8(66.66 \%)$ & $98.585 \pm 5.998$ & 0.301 \\
$(N=12)$ & $\geq 34$ & $4(33.33 \%)$ & $94.83 \pm 4.587$ & \\
\hline
\end{tabular}

Table 3: MAP and severity of PE

\begin{tabular}{llll}
\hline Severity of $P E$ & $N$ & Mean MAP, $\mathrm{mm} \mathrm{Hg}$ & p value \\
\hline PE without severe features & 6 & $97.22 \pm 7.03$ & 0.950 \\
PE with severe features & 6 & $97.45 \pm 4.60$ & \\
\hline
\end{tabular}

Table 4: *Diagnostic accuracy of MAP for GH and PE

\begin{tabular}{lll}
\hline Parameter & GH (\%) & PE (\%) \\
\hline Sensitivity & 84.2 & 83.3 \\
Specificity & 84.9 & 97.2 \\
NPV & 98.8 & 99.3 \\
PPV & 27.1 & 55.6 \\
Accuracy & 84.9 & 96.6 \\
\hline
\end{tabular}

${ }^{*}$ Using cutoffs from ROC, the MAP was 89.17 and $93.67 \mathrm{~mm} \mathrm{Hg}$ for GH and $\mathrm{PE}$, respectively

The sensitivity and specificity and NPV of MAP for diagnosing $\mathrm{GH}$ and PE were quite high in our study (Table 4), implying women who have MAP less than the cut-off values ( 89.17 for $\mathrm{GH}$ and $93.67 \mathrm{~mm} \mathrm{Hg}$ for $\mathrm{PE}$ ) in their second trimester of pregnancy are unlikely to develop GH or /PE later in the pregnancy. PPV of 27.1 and $55.6 \%$ was observed in our study for predicting the $\mathrm{GH}$ and $\mathrm{PE}$, respectively, which implies that the role of predicting the development of GH and PE with a higher MAP was moderate. However, it can be highly useful for predicting the low-risk pregnancy in terms of pregnancy hypertension because of their high NPV. Thus, the measurement of mid-trimester MAP can be used to triage low-risk women for developing pregnancy hypertension, who can be delivered in a low-resource setting.

A high specificity (91.4\%) and NPV (86.8\%) and moderate PPV (56.6\%) similar to our study were reported by Upadhyay et al., though a low sensitivity (44.7\%) was reported by her. ${ }^{17}$ Miller $^{14}$ reported that MAP greater than $88 \mathrm{~mm} \mathrm{Hg}$ predicted PE with a sensitivity of 0.78 and a specificity of 0.63 .

Some studies have demonstrated a higher predictive power when MAP is measured during the first trimester of pregnancy, ${ }^{18,19}$ particularly when combined with other maternal factors. However, a cohort of more than 70,000 pregnant women found a similar detection rate of $\mathrm{PE}$, with MAP measurement in the first and second trimesters of gestation. ${ }^{20}$ Performance of screening for PE by MAP has been the best when measurements are taken at both 11-13 and 20-24 weeks of gestation than at only one of these gestational ranges. $^{18}$

\section{Limitations}

Women at high risk of developing pregnancy hypertension if detected in the first trimester could have been subjected to prophylactic intervention by aspirin. As women were enrolled in the second trimester in our study, this opportunity of primary prevention was not available to our patients, hence, representing a limitation of our study. However, the prediction of GH and PE in mid-trimester can still redefine pregnancy, including the frequency of ANC visits, addressing time, method, and place of delivery. ${ }^{20,21}$

Another weakness of our study was that obesity was not an exclusion factor. MAP is known to be dependent on weight. ${ }^{22}$ However, an appropriate size cuff for each patient was selected for BP measurement. Therefore, future studies should be conducted to analyze the mean arterial blood pressure in combination with other factors, to obtain a predictive algorithm for PE.

\section{Conclusion}

We conclude from the results of our study that MAP in the second trimester of pregnancy has a strong association with the development of GH or PE in later pregnancy. The cutoff of midtrimester MAP greater than $89.7 \mathrm{~mm} \mathrm{Hg}$ can be a useful parameter to predict the development of GH and PE. These women should be considered high risk and should be delivered in a healthcare facility where a neonatal intensive care unit is available. MAP less than 89.7 $\mathrm{mmHg}$ can be used as a negative predictor for the development of $\mathrm{GH}$ or $\mathrm{PE}$, and these women may be considered low risk and can be delivered at primary or secondary level healthcare facilities. Thus, second trimester MAP can be used for risk triaging in women with regard to the development of pregnancy hypertension.

\section{Clinical Significance}

Mid-trimester MAP is a very good parameter for the prediction of $\mathrm{GH}$ and $\mathrm{PE}$. It should be routinely used for risk triaging. Low-risk women who are less likely to develop GH or PE, therefore, can be delivered at primary or secondary level healthcare facilities.

\section{References}

1. Jeyabalan A. Epidemiology of pre-eclampsia: impact of obesity. Nutr Rev 2013;71 Suppl. 1(0 1):S18-S25. DOI: 10.1111/nure.12055. PMID: 24147919; PMCID: PMC3871181.

2. Duley L. The global impact of pre-eclampsia and eclampsia. Semin Perinatol 2009;33(3):130-137. DOI: 10.1053/j.semperi.2009.02.010.

3. Kumari S, Gupta M, Vardan H, et al. NT pro-BNP: a biochemical marker of maternal complications in pre-eclampsia. JCDR 2017;11(11):QC12QC16. DOI: 10.7860/JCDR/2017/28713.10855.

4. Shanker O, Saini V, Gupta M. Stillbirths: incidence, causes and surrogate markers of intrapartum and antepartum fetal deaths. Int J Inn Res Med Sci 2020;5(08):289-295. DOI: 10.23958/ijirms/vol05-i08/927.

5. Conde-Agudelo A, Villar J, Lindheimer M. World Health Organization systematic review of screening tests for pre-eclampsia. Obstet Gynecol 2004;104(6):1367-1391. DOI: 10.1097/01.AOG.0000147599.47713.5d [Erratum in: Obstet Gynecol 2005;106:869].

6. Conde-Agudelo A, Romero R, Lindheimer M. Tests to predict preeclampsia. In: Lindheimer MD, Roberts JM, Cunningham FG, editors. Chesley's hypertensive disorders of pregnancy. 3rd ed. San Diego, CA: Elsevier; 2009 [in press].

7. Meads CA, Cnossen JS, Meher S, et al. Methods of prediction and prevention of pre-eclampsia: systematic reviews of accuracy and effectiveness literature with economic modeling. Health Technol Assess 2008;12(6):iii-iv1-270. DOI: 10.3310/hta12060.

8. Ayala DE, Hermida RC, Mojon A, et al. Blood pressure variability during gestation in healthy and complicated pregnancies. Hypertension 1997;30(3):611-618. DOI: 10.1161/01.HYP.30.3.611.

9. Poon LC, Nicolaides KH. Early prediction of pre-eclampsia. Obstet Gynecol Int 2014;2014:297397. DOI: 10.1155/2014/297397.

10. Roberts JM, August PA, Bakris G, et al. Hypertension in pregnancy: executive summary. Obstet Gynecol 2013;122(5):1122-1131. DOI: 10.1097/01.AOG.0000437382.03963.88. 
11. Umegbolu El, Ogamba JO. Incidence of gestational hypertension among pregnant women (2006-2015) in Enugu State, Southeast Nigeria: a retrospective study. Int J Community Med Public Health 2017;4(2):357-362. DOI: 10.18203/2394-6040.ijcmph20170255.

12. Rajesh A, Suresh A, Muralidharan V. Serum lipid profile in second trimester as a predictor of gestational hypertension. J South Asian Feder Obst Gynae 2020;12(1):23-26. DOI: 10.5005/ jp-journals-10006-1750.

13. Dawle SS, Bhalerao AV. Prediction of pregnancy-induced hypertension by maternal serum beta human chorionic gonadotropin levels in early second trimester of pregnancy. $J$ South Asian Feder Obst Gynae 2018;10(2):104-109. DOI: 10.5005/ jp-journals-10006-1570.

14. Miller RS, Rudra CB, Williams MA. First-trimester mean arterial pressure and risk of pre-eclampsia. Am J Hypertens 2007;20(5):573578. DOI: 10.1016/j.amjhyper.2006.12.012.

15. Mayrink J, Souza RT, Feitosa FE, et al. Mean arterial blood pressure: potential predictive tool for pre-eclampsia in a cohort of healthy nulliparous pregnant women. BMC Pregnancy Childbirth 2019;19(1):460. DOI: 10.1186/s12884-019-2580-4.

16. Cnossen JS, Vollebregt KC, de Vrieze N, et al. Accuracy of mean arterial pressure and blood pressure measurements in predicting pre-eclampsia: systematic review and meta-analysis. BMJ 2008;336(7653):1117-1120. DOI: 10.1136/bmj.39540.522049.BE.

17. Upadhyay A, Dayal M. Prediction of pre-eclampsia by mean arterial pressure and waist circumference. New Indian J OBGYN 2019;6(1):2427. DOI: 10.21276/obgyn.2019.6.1.6.

18. Poon LC, Syngelaki A, Akolekar R, et al. Combined screening for preeclampsia and small for gestational age at 11-13 weeks. Fetal Diagn Ther 2013;33(1):16-27. DOI: 10.1159/000341712.

19. Gasse C, Boutin A, Coté $M$, et al. First-trimester mean arterial blood pressure and the risk of pre-eclampsia: the great obstetrical syndromes (GOS) study. Pregnancy Hypertens 2018;12:178-182. DOI: 10.1016/j.preghy.2017.11.005.

20. Tayyar A, Krithinakis K, Wright A, et al. Mean arterial pressure at 12, 22, 32 and 36 weeks' gestation in screening for pre-eclampsia. Ultrasound Obstet Gynecol 2016;47(5):573-579. DOI: 10.1002/uog.15815.

21. Ghi T, Dall'Asta A, Valensise H. Antenatal care of pre-eclampsia: from the inverted pyramid to the arrow model? Fetal Diagn Ther 2018;44(2):81-84. DOI: 10.1159/000490047.

22. Wright $A$, Wright $D$, Ispas $C A$, et al. Mean arterial pressure in the three trimesters of pregnancy: effects of maternal characteristics and medical history. Ultrasound Obstet Gynecol 2015;45(6):698-706. DOI: 10.1002/uog.14783. 